\title{
Modeling radiation damage to pixel sensors in the ATLAS detector
}

\author{
A. Ducourthial, ${ }^{a, 1}$ on behalf of the ATLAS collaboration \\ ${ }^{a}$ Laboratoire de Physique Nucleaire et de Hautes Energies, LPNHE, \\ 4 place Jussieu, Paris, France \\ E-mail: audrey.ducourthial@cern.ch
}

ABSTRACT: Silicon pixel detectors are at the core of the current and planned upgrade of the ATLAS detector at the Large Hadron Collider (LHC). As the closest detector component to the interaction point, these detectors will be subject to a significant amount of radiation over their lifetime: prior to the High-Luminosity LHC (HL-LHC), the innermost layers will receive a fluence in excess of $10^{15} \mathrm{n}_{e q} / \mathrm{cm}^{2}$ and the HL-LHC detector upgrades must cope with an order of magnitude higher fluence integrated over their lifetimes. Simulating radiation damage is essential in order to make accurate predictions for current and future detector performance that will enable searches for new particles and forces as well as precision measurements of Standard Model particles such as the Higgs boson. We present a digitization model that includes radiation damage effects on the ATLAS pixel sensors for the first time. In addition to thoroughly describing the setup, we present first predictions for basic pixel cluster properties alongside early studies with LHC Run 2 proton-proton collision data.

KEYwORDS: Detector modeling and simulations I, Detector modelling and simulations II, Radiationhard detectors

\footnotetext{
${ }^{1}$ Corresponding author.
} 


\section{Contents}

1 The ATLAS inner detector 1

1.1 Planar pixel sensors 2

1.2 3D pixel sensors 2

2 Modeling radiation damages in pixel silicon sensors of the ATLAS inner tracker 3

2.1 Charge carriers dynamics in silicon pixel sensors 3

2.2 Radiation damage in silicon sensors 4

2.3 Modeling radiation damage 4

2.3.1 Determination of the fluence 4

2.3.2 Electric fields 5

2.3.3 Charge chunking 6

2.3.4 Ramo potential and Induced Charge 6

$\begin{array}{lll}3 & \text { Validation of the model } & 7\end{array}$

$\begin{array}{ll}3.1 & \text { Charge collection efficiency }\end{array}$

4 Conclusion $\quad 8$

\section{The ATLAS inner detector}

The ATLAS [1] inner detector [2,3] is dedicated to the study of charged particles tracks. It is composed of three sub-detectors including a pixel detector, the closest to the beam pipe, which is subdivised into a barrel part in the center and two endcap parts at each end of the detector. The barrel pixel detector is now running with 4 pixel layers, whose distance to the beam are documented in Figure 1. The first layer, namely the IBL (Insertable B Layer) [4] aims to distinguish tracks from $B$ hadrons decays, which is determinant for many physics analyses. Its proximity to the interaction point makes this detector particularly exposed to radiation damage. The three outer layers and $75 \%$ of the IBL sensing area are planar pixel sensors. The remaining areas at the extremity of the staves consist of module based with the new 3D technology [5]. Due to a harsh radiation environment, it is necessary to implement a modeling of radiation damage, to account for these effects in the simulations. The radiation damage digitizer will be essential during the data taking phase of ATLAS in the conditions of the high luminosity LHC (HL-LHC). In the following, the physics processes and the validation of the pixel radiation damage modeling that are being incorporated into the ATLAS simulation are documented. An article with a more detailed discussion is in preparation. 


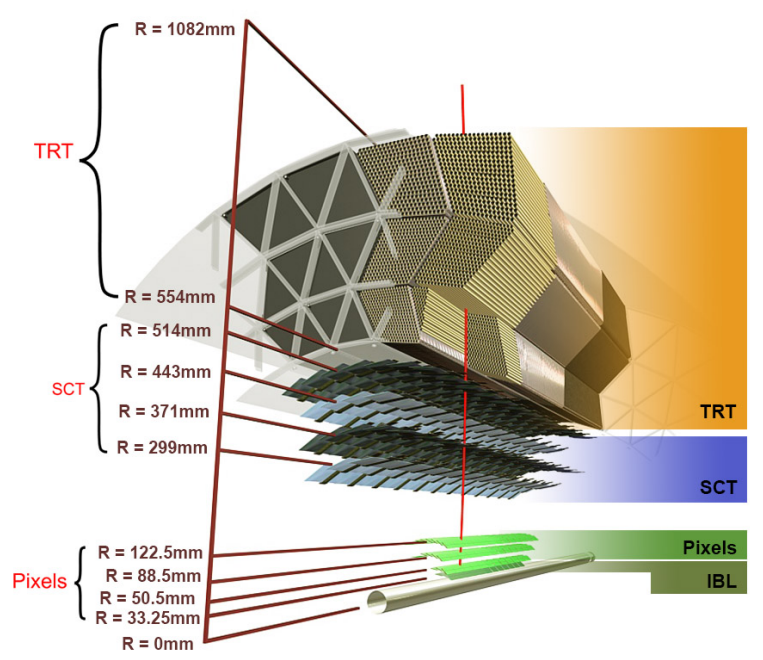

Figure 1. Inner ATLAS detector with Run 2 layout

\subsection{Planar pixel sensors}

The large hadron fluence cumulated so far and accelerated by the good performance of the LHC motivates to model the radiation damage induced effects in the pixel sensors. ATLAS planar pixel sensors are made of a silicon bulk slightly n-doped on top of which is deposited a thin layer of n-doped silicon implant. The bulk thickness is $200 \mu \mathrm{m}$ for the IBL and $250 \mu \mathrm{m}$ for the other layers. The top implant is highly segmented into pixels with a dimension of $400 \mu \mathrm{m} \times 50 \mu \mathrm{m}$ for the outer layers and of $250 \mu \mathrm{m} \times 50 \mu \mathrm{m}$ for the IBL.
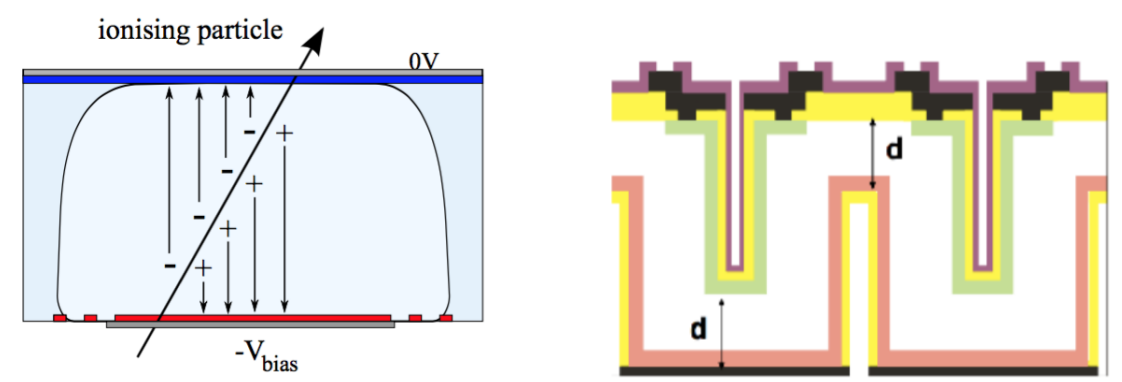

Figure 2. Planar pixel sensors (left) and 3D pixel sensors (right) [4] .

\subsection{D pixel sensors}

3D sensors consists of heavily doped pillars passing through the whole thickness of the sensor p-type bulk. The pitch is the same of the IBL planar sensor one $(250 \mu \mathrm{m} \times 50 \mu \mathrm{m})$ and the thickness is $230 \mu \mathrm{m}$. This technology is now known to be radiation hard with the main advantage of short collected distance allowing to operate with relatively low supply voltage of less than $200 \mathrm{~V}$. 


\section{Modeling radiation damages in pixel silicon sensors of the ATLAS inner tracker}

Modeling radiation damages involves a good understanding of the dynamics of charge carriers inside the sensor and the various mechanisms of radiation damage and their effects in the silicon. The first and second parts of this section document those aspects. A third part describes the various inputs and processes that are used in the digitizer.

\subsection{Charge carriers dynamics in silicon pixel sensors}

Once the sensor is depleted, the electrons and holes created by the passage of a MIP (Minimum Ionizing Particle) move according to several contributions:

- Drift: Free charge carriers follow the electrical field lines created due to the bias voltage.

- Diffusion: Thermal diffusion inside the sensor

- Lorentz angle: in the presence of the $2 \mathrm{~T}$ magnetic field, free charge carriers move with an angle compared to the electrical field $(E)$ direction called the Lorentz angle which is $\tan \theta_{L}=r \mu(E) B$ with $\mathrm{r}$ the Hall scattering factor, $\mu(E)$ the mobility and $B$ the magnetic field. Due to the vertical orientation of their electrodes, $3 \mathrm{D}$ sensors are quite insensitive to this effect.

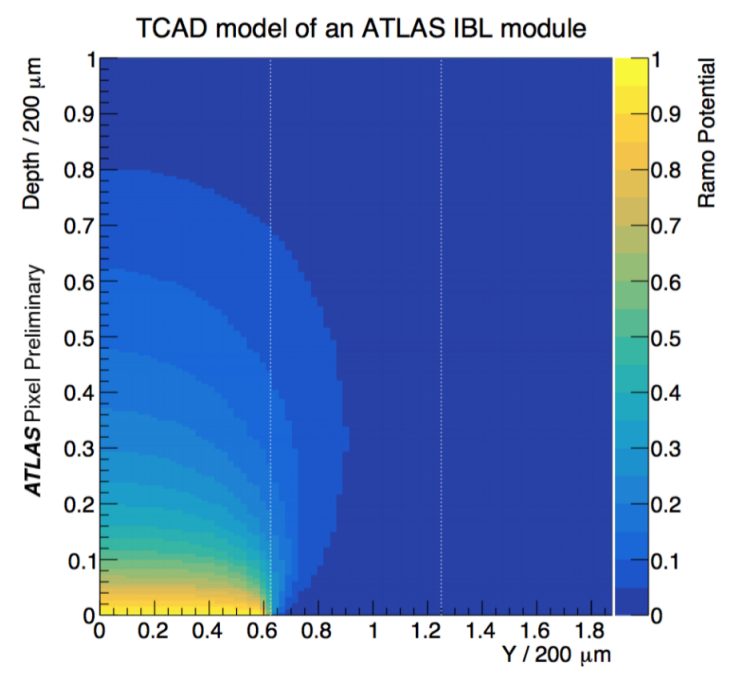

Figure 3. Ramo potential in one IBL planar sensor.

The movement of those charge carriers creates a signal in the electrode and the induced charge can be modeled by the Ramo potential [6]: the amount of induced charge is the particle charge multiplied by the difference between the Ramo potential at its starting point and its ending point. Figure 3 shows the simulated Ramo potential in an IBL planar module. One interesting feature is that a significant amount of charge can be induced in neighboring pixels (up to 20\%-30\%). 


\subsection{Radiation damage in silicon sensors}

The radiation damage digitizer focus on modeling the 2 main effects of radiation damages [7] produced in the silicon bulk by non ionizing particles:

- Charge Trapping: Due to the presence of defects in the bulk resulting in the creation of new states into the band gap, some electrons or holes can be trapped. Hence a loss of charge collection efficiency with the fluence is observed.

- Deformations in the electric field: Defects created by radiation damage can be charged. This charge changes the electrical field distribution in the sensor. This leads to type inversion of $\mathrm{n}$-type bulk into p-type bulk after a certain amount of fluence $\left(10^{13}-10^{14} \mathrm{n}_{e q} / \mathrm{cm}^{2}\right)$ and to a steady increase of the full depletion voltage after type inversion. The IBL underwent type inversion in 2015: before inversion, depletion grows from the backside towards the pixel implant; after inversion, the depletion grows from the pixel implant to the backside.

After stopping the exposure to particle fluence, it is observed for a limited time period a decrease of the effective doping concentration which is associated to a beneficial annealing. When exceeding a certain time the re-association of the trapping centers results in increasing the effective doping concentration which is called reversed annealing. The two processes are time and temperature dependent. Effects of such processes can be slowed down significantly below a certain temperature (around or below $0^{\circ}$ ).

\subsection{Modeling radiation damage}

Modeling radiation damage in pixel sensors requires various inputs and steps which are going to be described in the following. Prior to the digitization, it is necessary to collect information on the detector geometry and the fluence to which it is exposed. Then the input to the digitizer is an energy deposition in the sensor corresponding to the crossing of a charged particle (e.g from GEANT 4 [8]). Another important input is the electric field map expected in such sensors after irradiation, the Hamburg model is used to account for annealing; the electrical field is predicted using Technology Computed Aided Design (TCAD) simulation. Ionized charged are then undergo to the various dynamical components (drift, diffusion, Lorentz angle). Another ingredient in the digitization is the chunking of charges as it is computationally too heavy to drift each electron and hole. Finally the induced charge including the component from the trapped chunks is evaluated using a Ramo weighting potential, including neighboring pixels. The charge is then converted into time-over-threshold; clusters are formed by grouping neighboring pixels, and tracks reconstruction follows.

\subsubsection{Determination of the fluence}

The fluence is determined from the integrated luminosity. The FLUKA framework $[9,10]$ is used to estimate the conversion factor between integrated luminosity and the fluence. The fluence systematic uncertainties are evaluated by comparing the leakage current extracted from the fluence and the real leakage current which is precisely measured. A $15 \%$ inaccuracy is estimated due to the systematic uncertainty of the fluence calculation. 


\subsubsection{Electric fields}

The radiation-induced states in the silicon band gap affect the electric field in the pixel cells by altering the electric field in the bulk. So it is necessary to produce look-up tables of the electric field both for planar and 3D sensors and under various fluence conditions. Those simulations are done using Silvaco [11] or Synopsis [12] TCAD software. Thanks to geometrical symmetries of the pixel cells it is possible to simulate only a part of it. The charge collection mechanism is different for planar and 3D sensors, so that two different setups are implemented which are going to be discussed in what follows.
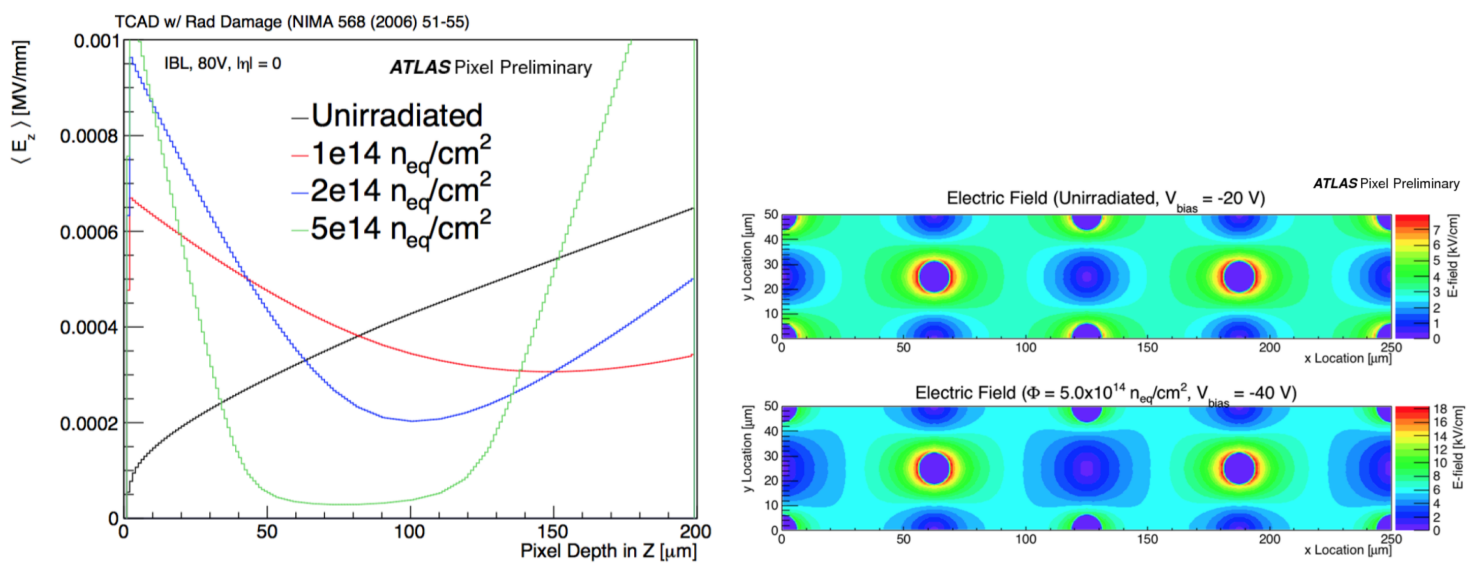

Figure 4. Electrical field in planar sensors (left) and 3D pixel sensors (right). For 3D sensor, the top plot is the electrical field before irradiation at $20 \mathrm{~V}$ and the bottom plot after receiving a fluence of $5 \times 10^{14} \mathrm{n}_{e q} / \mathrm{cm}^{2}$ at $40 \mathrm{~V}$

Planar sensors: The model used to describe the electric field in planar sensor is the Chiochia [13] model which contains two defect states: one acceptor trap and one donor trap with activation energies set to $E_{c}-0.52 \mathrm{eV}$ and $E_{v}+0.48 \mathrm{eV}$ (with $E_{c}$ the energy of conduction band and $E_{v}$ the energy of the valence band). Other parameters of importance for this model are the capture cross section for acceptors and donors for holes and electrons, and the donor and acceptor concentration. Figure 4 shows on its left a field profile of one IBL planar pixel sensor at $80 \mathrm{~V}$, in the bulk thickness dimension simulated for 4 different fluences. When the sensor is unirradiated, the field is quasi linear: the depletion grows from the back side (where one can find the maximum value of the field) toward the pixel implant. After type inversion, the field maximum is on the opposite side of the sensor. With increasing fluence, there is a minimum in the electric field in the center of the sensor. For a fluence of $5 \times 10^{14} \mathrm{n}_{e q} / \mathrm{cm}^{2}$ for $80 \mathrm{~V}$ (under depletion regime), this minimum is broad and occupies nearly a third of the sensor.

3D sensors: Unlike planar pixel sensors, electrons and holes in 3D sensors drift laterally and not through the bulk depth. Radiation damage effects for the 3D sensor are implemented in the Perugia [14] model with the Synopsys [12] TCAD package. Just one-eight of the sensor is simulated to take advantage of the symmetry within the pixel. In the Perugia model [14], there are two acceptors and one donor defects states, with activation energies given by $E_{c}-0.42 \mathrm{eV}, E_{c}-0.46 \mathrm{eV}$, and $E v+0.36 \mathrm{eV}$, respectively. The field distribution in the $\mathrm{XY}$ plan is presented in the right of Figure 
4 for one pixel cell of unirradiated sensor (top) and irradiated at a fluence of $5 \times 10^{14} \mathrm{n}_{e q} / \mathrm{cm}^{2}$ at the bottom. The $n+$ and $p+$ implants are regions of no field due to their large doping.

Other models, such as the Petasecca model [15] were considered to describe planar sensors. An estimation of the uncertainties has been developed and will be available in the soon-coming article.

\subsubsection{Charge chunking}

In the pixel sensor, approximately 80 electron-hole pairs are generated per micron from the passage of a charge particle The digitization of so many charge carriers is computationally too heavy, so charge carriers are clusterized in chunks. Even if the mean cluster charge is similar if one considers chunks collection or single free charge carriers, the fluctuations in the induced charge can be over-estimated. So the fluctuations have to be unsmeared: the mean collected charge is also computed and $Q \rightarrow<Q>+\frac{1}{\sqrt{n}}(Q-<Q>)$ has the correct fluctuation size, $\mathrm{n}$ being the number of fundamental charges represented by one chunk. Figure 5 shows the distribution of charge collection vs time to electrode and illustrates the effect of the unsmearing. The left plot is for 10 chunks, the mid one for 100 and the right one for 1000. The blue and green contributions are respectively collected and induced charge, before the corrections; the red and pink contributions are collected and induced charge after the unsmearing. The effect of unsmearing is really striking in the middle and left plots, where the distribution is much more broader before the corrections.
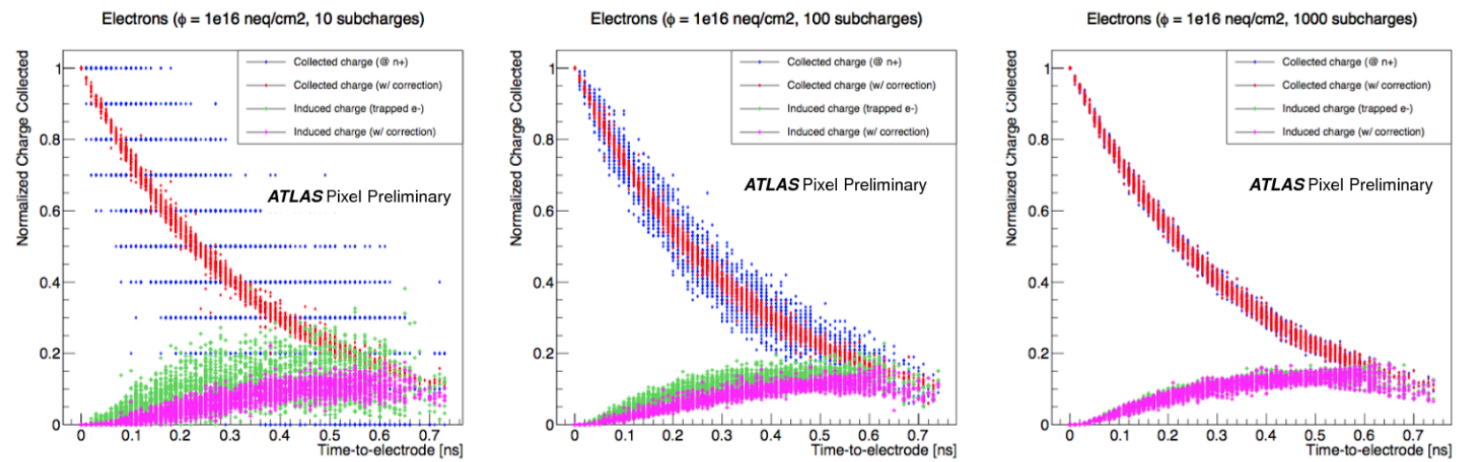

Figure 5. Charge chunking unsmearing. This figure shows the normalized charge collected vs time to electrode. The red and blue curve are the collected charge respectively with/without unsmearing. The pink and green curve are the induced charge by trapped carriers respectively with/without unsmearing. The three plots are considering different numbers of subcharges (chunks): the left plot shows results for 10 chunks, the middle one for 100 chunks and the right one for 1000 chunks.

\subsubsection{Ramo potential and Induced Charge}

Even if charges are trapped, they still induce a signal which can be evaluated using the Ramo potential [6]. The left part of Figure 6 shows this effect: three electrons are created in the up left corner and then drift following electrical lines. At some point they are trapped but induce a charge as shown in the figure. The right part of Figure 6 shows the weighting potential value in all the XY plan for 2 simulated 3D electrodes. 

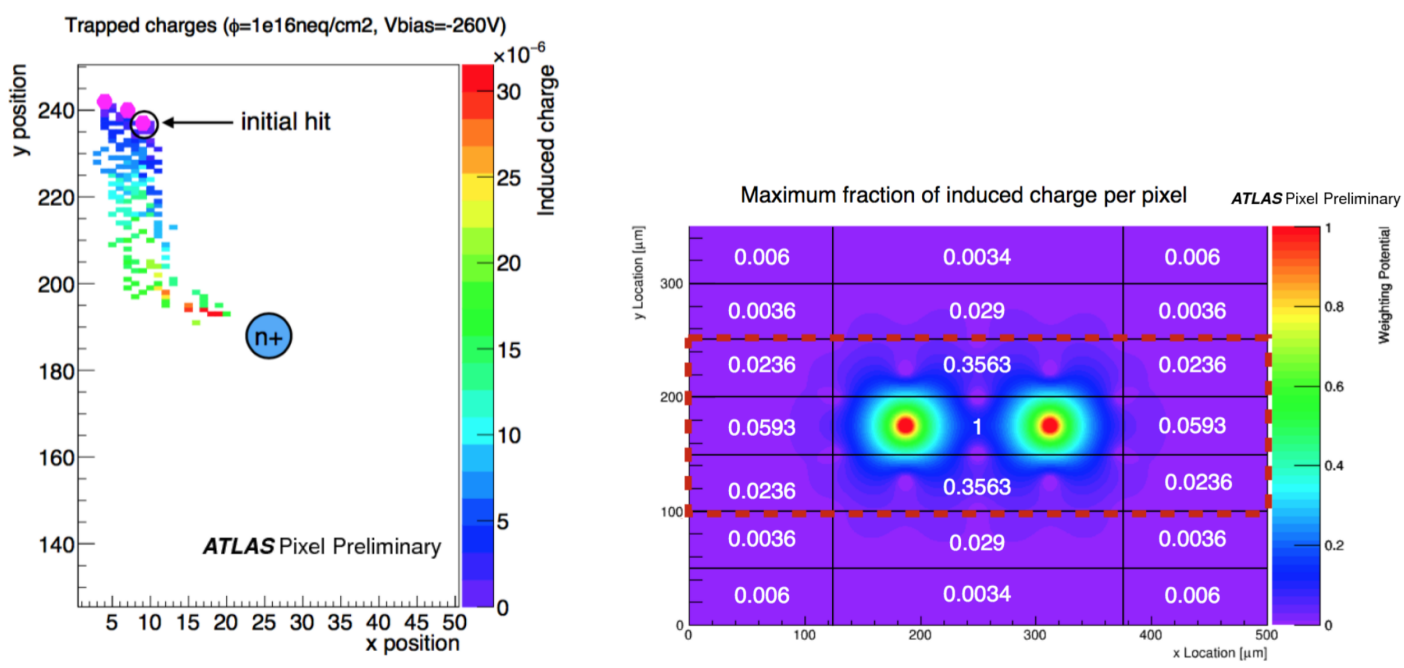

Figure 6. Trapping effect on induced charge in 3D sensor. The left plot shows the induced charge at trap location for 3 electrons created in the upper half part. The right plot is a 2D map in the XY plan of the weighting potential for 2 simulated 3D columns.

\section{Validation of the model}

The accuracy of the radiation damage modeling can be determined by comparing the charge collection efficiency between collision data and simulated data from the digitizer.

\subsection{Charge collection efficiency}

Figure 7 shows a comparison of the charge collection efficiency between IBL data collected during 2015 and 2016, with a Bias Voltage of $80 \mathrm{~V}$ and $150 \mathrm{~V}$ and simulated datasets implemented thanks to the AllPix [16] digitizer which is a lighter version of the full ATLAS simulation framework.

The fraction of collected charge is the ratio of charge collected by an irradiated device and of the charge collected by over-depleted unirradiated devices.

The uncertainties accounts for the following phenomena:

- Uncertainty of $3 \%$ on the luminosity for the data (horizontal error bars)

- Uncertainty of $15 \%$ on the luminosity for the simulated data due to the fluence to luminosity conversion (horizontal error bars)

- Vertical error bars due to the uncertainty on radiation model parameters such as energy of defects, concentration and capture cross section and trapping constant.

- Vertical error bars in data due to the drift in the tuning of the modules.

The level of agreement between data and simulation is satisfactory. 


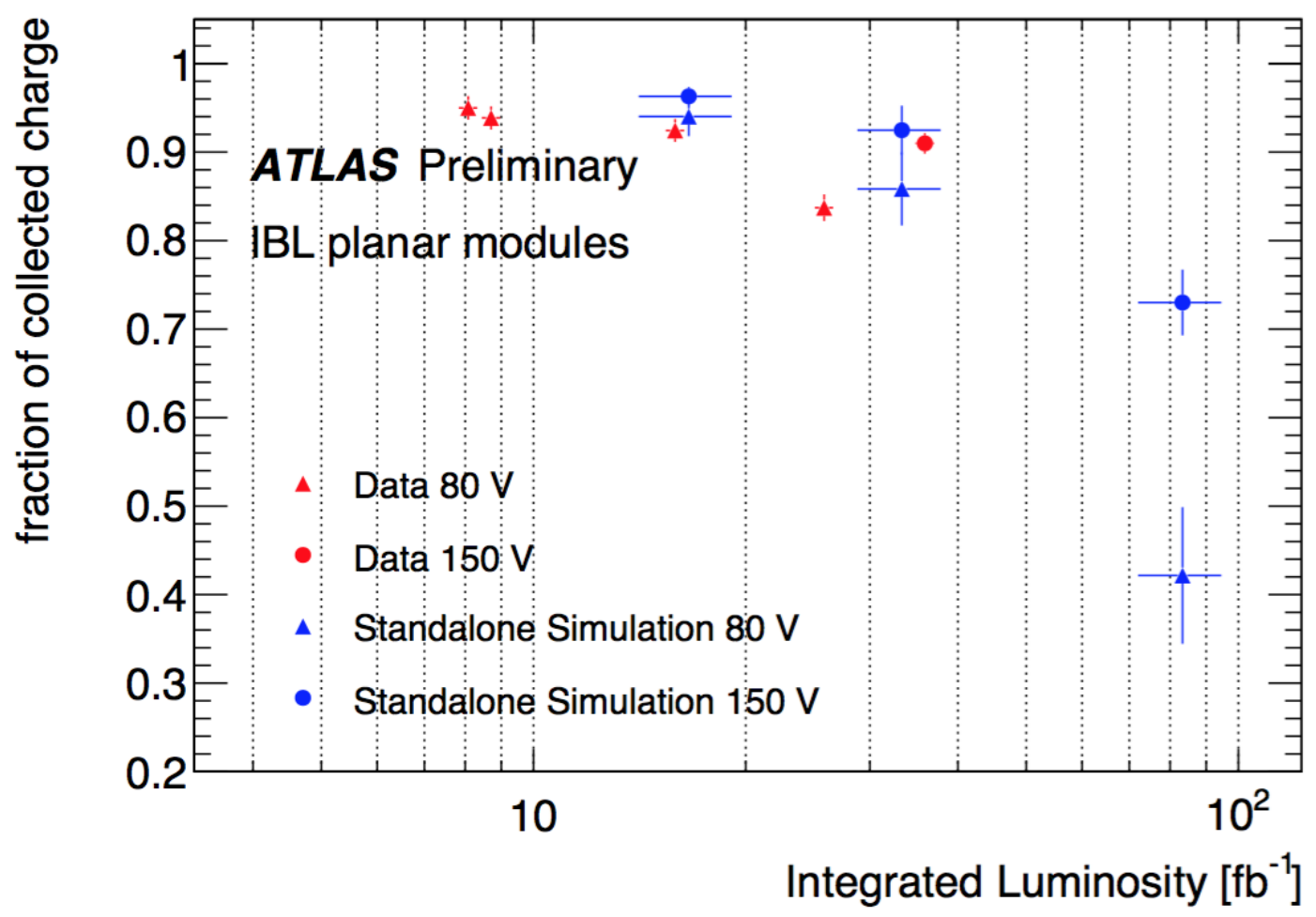

Figure 7. Charge collection efficiency comparison between data and simulation [17]

\section{Conclusion}

A digitization model for ATLAS planar and 3D pixel sensors that includes radiation damage effects has been presented. By providing a better matching between MC and data, it aims to create good prediction towards the next data taking period (Run 3) but also to make important design decisions for the upgraded ATLAS detector that must survive the harsh HL-LHC radiation environment. In addition to describing the physics processes incorporated in the digitizer, the charge collection efficiency is studied using Run 2 data to show reasonable agreement between the simulation and the observations. A more detailed publication will be available soon.

\section{References}

[1] ATLAS Collaboration, The ATLAS Experiment at the CERN Large Hadron Collider, JINST 3 (2008) S08003

[2] ATLAS Collaboration, The ATLAS Inner Detector commissioning and calibration, Eur. Phys. J. C70 (2010) 787

[3] G. Aad et al., ATLAS pixel detector electronics and sensors, JINST 3 (2008) P07007

[4] ATLAS Collaboration, ATLAS Insertable B-Layer Technical Design Report, CERN-LHCC-2010-013. ATLAS-TDR-19, 2010, https://cds.cern.ch/record/1291633 
[5] S.I.Parker, C.J.Kenney, J.Segal, 3D - A proposed new architecture for solid-state radiation detectors, Nucl. Instr. and Meth. A395 (1997) 328

[6] S. Ramo, Currents Induced by Electron Motion, Proceedings of the IRE 27 (1939) 584

[7] M. Moll, Radiation damage in silicon particle detectors: Microscopic defects and macroscopic properties, PhD thesis: Hamburg U., 1999, URL:

http://www-library.desy.de/cgi-bin/showprep.pl?desy-thesis99-040

[8] S.Agostinelli et al, GEANT4: A Simulation toolkit, Nucl. Instrum. Meth. A506 (2003) 250

[9] G. Battistoni et al., The FLUKA code: Description and benchmarking, AIP Conf. Proc. 896 (2007) 31

[10] A Ferrari et al., FLUKA: A multi-particle transport code (program version 2005), CERN, 2005, URL: https://cds.cern.ch/record/898301

[11] Silvaco, http://www.silvaco.com, Santa Clara, California (USA)

[12] Synopsys, http://synopsys.com, Mountain View, California (USA)

[13] V. Chiochia et al., Simulation of Heavily Irradiated Silicon Pixel Sensors and Comparison With Test Beam Measurements, IEEE Trans on Nucl. Sc. 52 (2005) 1067

[14] F. Moscatelli et al., Combined Bulk and Surface Radiation Damage Effects at Very High Fluences in Silicon Detectors: Measurements and TCAD Simulations, IEEE Transactions on Nuclear Science 63 (2016) 2716

[15] M. Petasecca et al., Numerical Simulation of Radiation Damage Effects in p-Type and n-Type FZ Silicon Detectors, IEEE Transactions on Nuclear Science 53 (2006) 2971

[16] J.Idarraga and M. Benoit, Generic Geant4 implementation for pixels, The Allpix Simulation Framework (2006)

[17] ATLAS Collaboration, Charge Collection Efficiency as a function of integrated luminosity, https://atlas.web.cern.ch/Atlas/GROUPS/PHYSICS/PLOTS/PIX-2017-004/ 